\title{
Enhancing negative messages broadcasting with Meet-Table and TTL in VANET
}

\author{
Xiaolu Cheng ${ }^{2}$, Jiawei Mo ${ }^{1}$ and Baohua Huang ${ }^{1 *}$
}

\begin{abstract}
In VANET (vehicular ad hoc network), a negative message has an objective vehicle and describes the negative attributes of the vehicle. Broadcasting negative messages in VANET is essential to make the VANET secure for these messages are about the untrustworthy vehicles. The epidemic model distributes negative messages rapidly with unlimited broadcasting, but uncontrolled epidemic may cause flooding storm. $T L$ (time-to-live) can be used to control broadcasting, but it may heavily decrease the coverage of negative messages in VANET. Meet-Table is used with $T \mathrm{~L}$ to solve the problem. A Meet-Table is a data structure held by a vehicle to record vehicles it met. When a vehicle receives a message, it checks whether the message's objective vehicle is in its Meet-Table. If it is, the vehicle resets the $T L$ of the message to the maximum $T L$ to extend the transmission of the message. The negative message, coverage percentage and accurate coverage percentage of the negative message, and the Meet-Table are formally defined. The algorithm for broadcasting negative messages with Meet-Table and $T L \mathrm{~L}$ is given. The simulation results show that Meet-Table can increase the coverage and decrease the delay of negative messages. More importantly, the simulation results show that the coverage increased by Meet-Table is accurate.
\end{abstract}

Keywords: Negative message, Meet-Table, VANET, Broadcast, Epidemic

\section{Introduction}

As a hybrid network, VANET (vehicular ad hoc network) consists of three communication domains: in-vehicle domain, ad hoc domain, and infrastructure domain [1]. The in-vehicle domain consists of OBU (on-board unit) and AU (application unit) and is representing with a vehicle in this paper. The ad hoc domain consists of vehicles. The infrastructure domain consists of vehicles and RSUs (roadside units). Accordingly, the VANET in this paper is the hybrid network consisted of vehicles and RSUs.

VANET supports many transportation applications to improve safety, efficiency, convenience, etc. [2]. As we know, the most significant and attractive application of VANET is to improve safety [3]. In VANET, a vehicle can gather information about the traffic condition around itself to improve its safety. The safety-related information includes speed limit, work zone notification, curve

\footnotetext{
*Correspondence: bhhuang66@gxu.edu.cn

'School of Computer and Electronic Information, Guangxi University, 100

Daxue Road, Nanning 530004, Guangxi, China

Full list of author information is available at the end of the article
}

warning, and accident information. In fact, these are traditional safety-related information. With the development of VANET, the dangerous and untrustworthy vehicle identification information are going to play more important role in the safety applications. We call this type of information negative messages [4], as it is used to describe the negative sides of a vehicle.

Negative messages include but are not limited to dangerous driving, untrustworthy certificate, and blacklist. Compared to other information in VANET, a negative message has two significant properties: (1) it is used to describe a definite vehicle, and (2) it is cared by the vehicles which may encounter that vehicle. Accordingly, a negative message needs not be distributed to all vehicles in the VANET; instead, it should be distributed to a subset of vehicles that may encounter the vehicle which the negative message described.

Information management in VANET has been extensively studied [2], and there are a few works about distributing CRL (certificate revocation list) in VANET [5, 6], but as far as we know, there is no work that clearly presents

\section{Springer Open}

(c) The Author(s). 2018 Open Access This article is distributed under the terms of the Creative Commons Attribution 4.0 International License (http://creativecommons.org/licenses/by/4.0/), which permits unrestricted use, distribution, and reproduction in any medium, provided you give appropriate credit to the original author(s) and the source, provide a link to the Creative Commons license, and indicate if changes were made. 
the concept of negative message. In [5], VII (vehicle infrastructure integration) tries to distribute CRL to vehicles through RSU broadcasting. This method required a very large number of RSUs and high cost. In [6], Haas et al. try to propagate CRL in an epidemic fashion. The epidemic method can distribute CRL to all vehicles with less number of RSU and less time, but it requires a large storage and communication capacity in VANET. In our previous work, we propose Meet-Table to optimize CRL propagation in VANET [7] and a scheme base on Meet-Table and cloud computing to distribute negative messages in VANET [4]. In this paper, we apply the Meet-Table and TTL (time-tolive) together to enhance negative message broadcasting in VANET.

The epidemic method [6] can rapidly distribute messages in VANET through broadcasting. But in a large VANET, if there is no limitation of broadcasting, flooding storm may destroy the availability of it. TTL can be used to restrict flooding [8]. A large TTL can hardly restrict flooding, while a small TTL will sharply decrease the coverage of the message.

As humans' movement follows simple reproducible patterns [9] and the trajectories of vehicles are a part of humans' movement, trajectories of vehicles are certainly reproducible. In other words, a vehicle may encounter the approximately the same set of RSUs and vehicles every day. A Meet-Table of a vehicle is used to record vehicles the vehicle met. Therefore, we can reset the TTL of a message during the message broadcasting with the help of Meet-Table. Using Meet-Table with TTL to broadcast negative message in a VANET, a balance between flooding control and message coverage can be easily achieved. Our major contributions in this paper include the following:

(1)We utilize Meet-Table to lead resetting of the TTL of message in negative message broadcasting in the VANET to achieve high availability and high coverage of the message.

(2)We illustrate the principle and give the algorithm of using Meet-Table with TTL in negative message broadcasting in VANET.

(3)We simulate Meet-Table with TTL in negative message broadcasting and give the results.

\section{Methods}

In this paper, we apply modeling and experiment to study the problem and the corresponding solution proposed above. Firstly, we formally define the negative message and give the calculation formula of possessing percentage and coverage percentage and accurate coverage percentage of the negative message. Based on the formal definition of these concepts, the evaluation criteria of distribution method of negative message are obtained.

Then, we model the Meet-Table and design the algorithm of broadcasting negative message in VANET utilizing Meet-Table and TTL. In order to illustrate the principle of enhancing negative message broadcasting with Meet-Table and TTL, a sample VANET having several vehicles is given and formally described.

Finally, we take experiments to simulate and evaluate the designed algorithm. We implement the algorithm in Matlab. The key parameters in the experiments are described in Section 5.

\section{Model of negative message in VANET}

With the development of the autonomous vehicle, more types of safety information are needed in VANET, for example, certificate, CRL, and the blacklist of untrustworthy vehicles. These types of information are used to describe vehicles' negative aspects, so we call them negative messages [4]. Firstly, we define the negative message formally.

Definition 1 A negative message is a negative description of a vehicle. Formally, negative message

$$
m \stackrel{\text { def }}{=}<o, d, C>
$$

In Formula 1, $m$ is a negative message, and it is a threetuple consisted by $v, d$, and $C$; $o$ is the objective vehicle of $m ; d$ is the data in the message describing $o$; and $C$ is a set of vehicles that concern the message $m$. A negative message uniquely binds to a vehicle. As the negative message is not useful for all vehicles in the VANET, it has a set of vehicles that care about it.

In VANET, we should process negative message $m$ in a way that (1) can push $m$ to all vehicle $v \in C$ as soon as possible; (2) for every vehicle $v \in C$, can get $m$ with high availability. General message dissemination methods in VANET try to distribute data to all vehicles. These methods are not very efficient and suitable for negative messages. For example, broadcasting CRL in a national wide VANET is not only unfeasible but also unnecessary [7]. For evaluating the method processing negative messages, we define possessing percentage, coverage percentage, and accurate coverage percentage in the following.

Definition 2 The possessing percentage of a negative message is the percent of vehicles possessing the message in all vehicles in VANET. Formally, coverage percentage

$$
r_{p}=\frac{|B|}{|V|}
$$

Definition 3 The coverage percentage of a negative message is the percent of vehicles possessing the message in vehicles concerning the message. Formally, coverage percentage 


$$
r_{c}=\frac{|B \cap C|}{|C|}=\frac{\sum_{b \in B}\left\{\begin{array}{l}
1, b \in C \\
0, b \notin C
\end{array}\right.}{|C|}
$$

Definition 4 The accurate coverage percentage of a negative message is the percent of vehicles concerning the message in vehicles possessing the message. Formally, accurate coverage percentage

$$
r_{a c}=\frac{|B \cap C|}{|B|}=\frac{\sum_{b \in B}\left\{\begin{array}{l}
1, b \in C \\
0, b \notin C
\end{array}\right.}{|B|}
$$

From Eqs. 2 to $4, B$ represents the set of vehicles that possess the negative message, $V$ denotes the set of all vehicles in VANET, and $C$ refers to a set of vehicles that concern the message.

In fact, the coverage percentage of a negative message represents the availability of the message, and the accurate coverage percentage of a message represents the efficiency of the distribution method. Naturally, we can give the evaluation criteria of distribution method of negative message.

Evaluation criteria 1: A good negative message distributing method should have both high coverage percentage and high accurate coverage percentage.

\section{Principle of enhancing negative message broadcasting with Meet-Table and TTL}

Meet-Table of a vehicle records vehicles the vehicle met in the past. We define Meet-Table formally, give a sample to illustrate the principle of using Meet-Table with TTL to enhance negative message broadcasting, and give the corresponding algorithms.

\subsection{Meet-Table}

In VANET, if we know $C$, which is the set of vehicles caring negative message $m$, we can accurately distribute $m$ to all vehicles in $C$. In fact, vehicles in $C$ are those vehicles that may encounter the objective vehicle of $m$. According to the reproducible moving patterns of human [9], we can assume that these vehicles met in the past may encounter one another in the future. So, we can record the vehicles passed a vehicle with a table, called Meet-Table. Formally, Meet-Table of $w$, a vehicle, can be defined as:

$$
\begin{aligned}
& T_{w}=\left\{p_{i} \mid 0 \leq i \leq n_{t_{w}}\right\} \\
& p_{i} \stackrel{\text { def }}{=}<v, t, c>, v \text { passed } w
\end{aligned}
$$

In Eq. 5 and Formula 6, $T_{w}$ is the Meet-Table generated by $w ; n_{T_{w}}$ is the number of elements in $T_{w} ; p_{i}$ is the $i$ th recorder in $T_{w}$, and it is a three-tuple consisted of $v, t$, and $c ; v$ is the vehicle passed $w c$ times by time $t$. For negative message $m$, if $\exists p_{i} \in T_{w}, p_{i} . v=m . o$, then $m$ should be broadcasted through $w$.

\subsection{Principle}

In order to illustrate the principle of enhancing negative message broadcasting with Meet-Table and TTL, we introduce a sample VANET. Figure 1 shows such a VANET.

In the VANET shown in Fig. 1,

$$
\begin{array}{r}
R=\{u 1\} \\
V=\{v 1, v 2, v 3, v 4, v 5, v 6\} \\
m 1 . o=v 1 \\
m 1 . C=\{v 2, v 3\} \\
T_{v 3}=\{<v 1, \ldots>, \ldots\} \\
T_{v 2}=\{<v 1, \ldots>, \ldots\} \\
T_{v 1}=\{<v 2, \ldots>,<v 3, \ldots>, \ldots\} \\
\mathrm{TTL}=3
\end{array}
$$

Where $R$ refers to the set of RSU. TTL is the maximum of total time to live of the negative message $m$.

From Formulas 7 to 14 and Fig. 1, we can see the enhancement of Meet-Table with TTL in negative messages broadcasting in VANET.

As shown in the bottom of Fig. 1, when TTL is used to control broadcasting, $m 1$ can only transmit to $v 6, v 5, v 4$, and $v 3 . v 2$ cares $m 1$, but it can not get the negative message

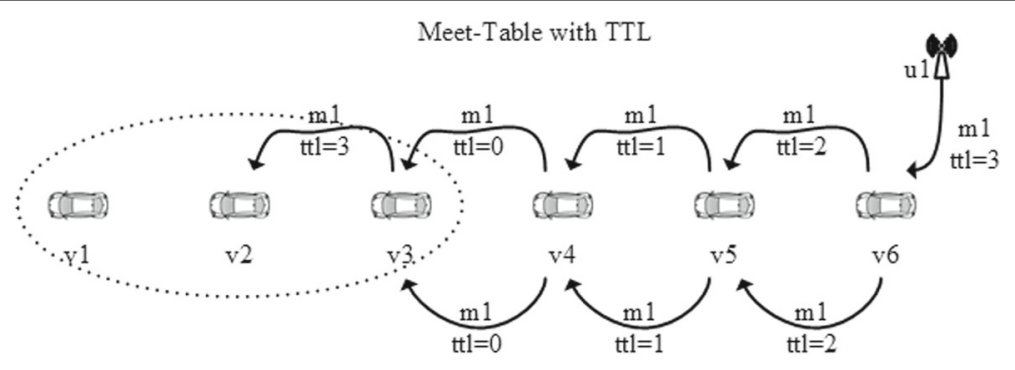

TTL

Fig. 1 Principle of enhancing negative message broadcasting with Meet-Table and $T \mathrm{TL}$ 
$m 1$ for the TTL of $m 1$ is decreased to 0 at $v 3$, before it is transmitted to $v 2$.

When Meet-Table is used with TTL, as shown in the top of Fig. $1, m 1$ 's TTL can be reset at $v 3$ for $m 1$ 's objective vehicle $v 1$ and $v 1$ is in $v 3$ 's Meet-Table. Consequently, $m 1$ can be continually transmitted to $v 2$.

\subsection{Algorithms}

Now, we can design the algorithm, namely AlgMeetTableTTL_Broadcast, to use the Meet-Table with TTL to enhance the negative messages broadcasting in VANET. For simplicity, we give the algorithm Broadcast_step first.

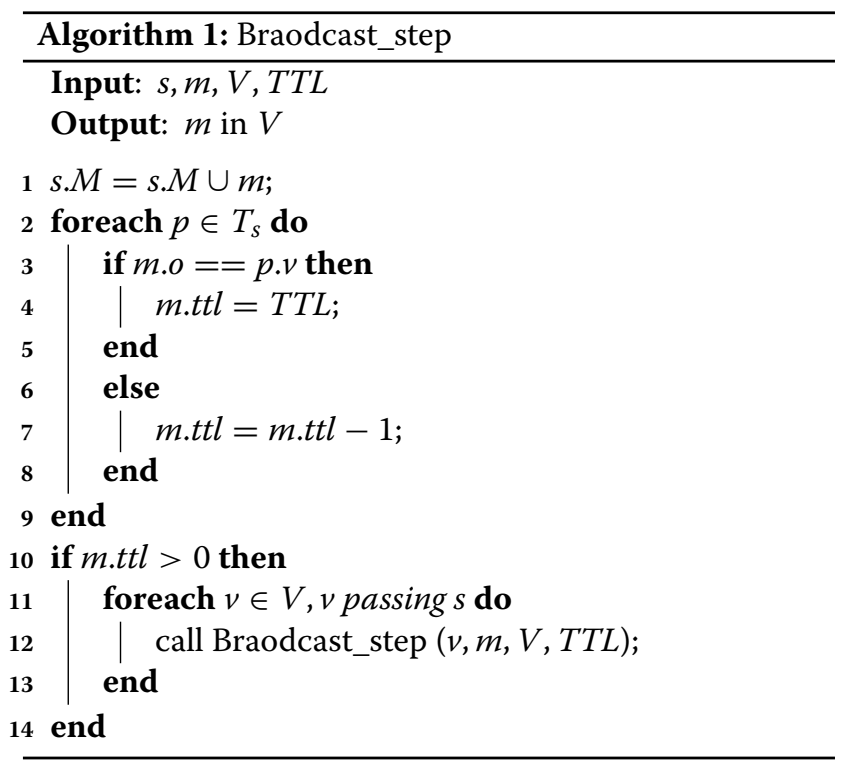

In Broadcast_step, $s$ is the vehicle as the start point of broadcasting. $m$ is the negative message waiting to be broadcasted. Obviously, this algorithm is recursive. It is called by itself and AlgMeetTableTTL_Broadcast.

AlgMeetTableTTL_Broadcast is the main algorithm. It takes $m$ and $V$ as inputs, generates a set of start points for broadcasting, sets $m$ 's TTL, then calls the algorithm Broadcast_step for all start points to fire the broadcasting.

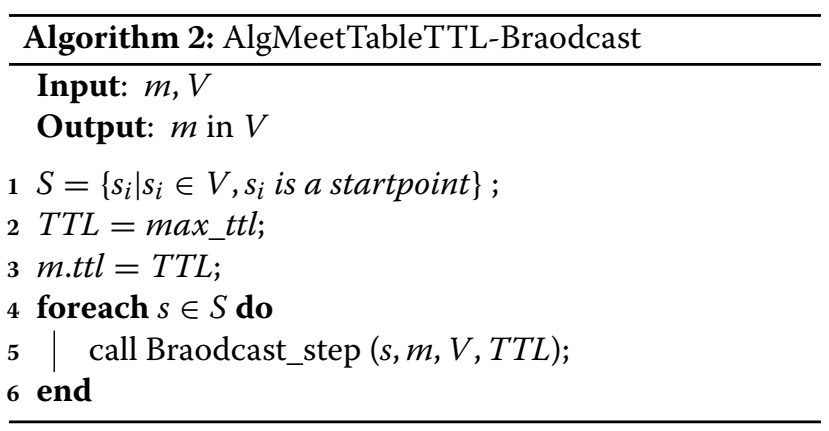

\section{Experiments}

To study the performance of Meet-Table with TTL for negative messages broadcasting in VANET, we do experiments by generating a simulation dataset and do simulations on the dataset. The experiment preparation and the experimental results are presented in this section.

\subsection{Experiment preparation}

Simulation of VANET can use a dataset of realistic traces of vehicles [10] or generate traces based on the map $[6,11]$. In fact, realistic trace dataset of numerous vehicles is very hard to get. The dataset used in [10] is realistic Taxi GPS traces from Shenzhen and Beijing, China, and San Francisco, USA. The total number of vehicles in this dataset is only about 13,000, and it contains only Taxi, no other types of vehicles. In addition, the time length of this dataset is no more than 3 days.

In order to evaluate the performance of the proposed scheme, we generated a dataset to simulate all vehicles in San Francisco, USA. The dataset was created based on the parameters shown in Table 1.

Figure 2 shows the percent of vehicles a vehicle met. The percent increases at first but keeps static after a certain point of time. This means that vehicles in the dataset have local attribute. In other words, the behavior of the vehicles is like what is demonstrated in [9]. So, the generated dataset is representative for the real dataset and adaptable to the simulation.

\subsection{Experimental results}

The typical methods used to distribute negative message, such as CRL, in VANET are RSU broadcasting [5] and epidemic [6]. In our previous work, we simulated RSU broadcasting, epidemic, and Meet-Table and found that epidemic is better than RSU broadcasting, so in this paper, we only compare the proposed method with epidemic.

Table 1 Parameters for generating simulation dataset

\begin{tabular}{|c|c|c|}
\hline Parameter & Value & Note \\
\hline Number of vehicles & 471388 & Total number of vehicles in [21]. \\
\hline Intersections & 7200 & $\begin{array}{l}\text { Estimated no. of intersections in } \\
\text { [21]. }\end{array}$ \\
\hline Length of road & 1741 km & Total length of road in [21]. \\
\hline Area & $121 \mathrm{~km}^{2}$ & Area-land in [21]. \\
\hline Mean travel time & $0.5 \mathrm{~h}$ & Mean travel time to work in [21]. \\
\hline Speed & $38.6 \mathrm{~km} / \mathrm{h}$ & $\begin{array}{l}\text { Average speed of commuter traffic } \\
\text { speeds in [22]. }\end{array}$ \\
\hline $\max V 2 \mathrm{~V}$ & $10 \mathrm{~m}$ & $\begin{array}{l}\text { Max communication distance of } \\
\text { vehicle to vehicle }\end{array}$ \\
\hline Start points & $0.0255 \%$ & \\
\hline$\pi \mathrm{T}$ & 6 & \\
\hline
\end{tabular}




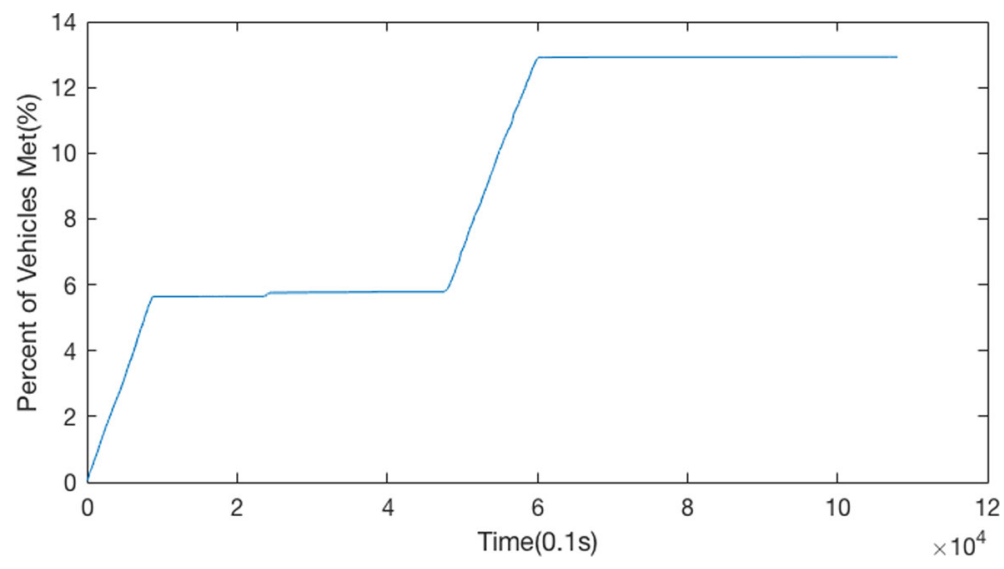

Fig. 2 Time vs. percent of vehicles met

We simulate broadcasting methods of epidemic without broadcasting control (shortly epidemic), epidemic with TTL, and Meet-Table (epidemic with TTL and MeetTable) on the generated dataset. The simulation results are shown in Figs. 3, 4, 5, and 6.

Figure 3 shows that the percent of vehicles possessing message increases with the simulation time. The epidemic has the highest and fastest increasing rate of the percent. The epidemic with TTL has the lowest and slowest increasing rate of the percent. The epidemic with TTL and Meet-Table has the middle percent and middle increasing rate of the percent. Obviously, TTL can sharply control the propagation of the message, and Meet-Table can mitigate the control of TTL.

Figure 4 shows that epidemic and epidemic with TTL and Meet-Table have similar coverage percent, but epidemic has a very low coverage percent. The function of Meet-Table with TTL in enhancing the distribution of negative message is demonstrated clearly.

Figure 5 shows that epidemic with TTL and MeetTable has high accurate coverage percent, but epidemic and epidemic with TTL have similarly low accurate coverage percent. It means that the enhancement of TTL with Meet-Table in propagation of negative message is accurate.

Figure 6 shows that epidemic with TTL has the highest delay time. Epidemic and epidemic with TTL and MeetTable have similarly lower delay time.

We summarize the simulation results in Table 2.

In Table 2, we can see that (1) Meet-Table with TTL increases the coverage of TTL, (2) Meet-Table with TTL has a higher accurate coverage percentage than epidemic with and without TTL have, (3) the delay of Meet-Table with TTL is as low as that of epidemic. So, Meet-Table with TTL can not only increase coverage but also accurately and timely increase coverage.

According to evaluation criteria 1, epidemic with MeetTable and TTL is better than epidemic with or without TTL for distributing negative messages in VANET.

\section{Discussion}

Improving the safety of traffic is the major goal of VANET. To achieve this goal, VANET must gather, process, and disseminate information, such as road condition, the

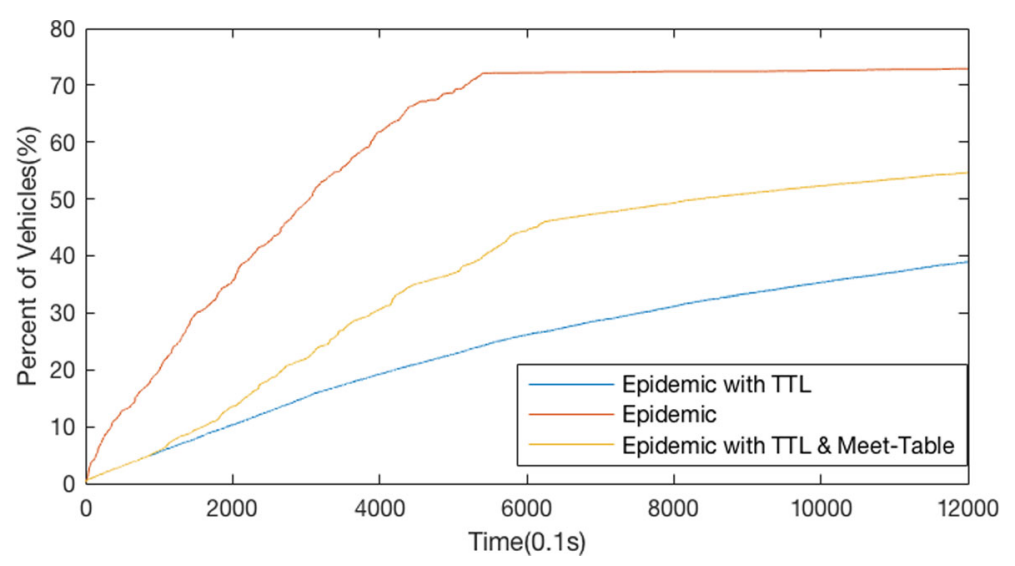

Fig. 3 Time vs. percent of vehicles possessing message 


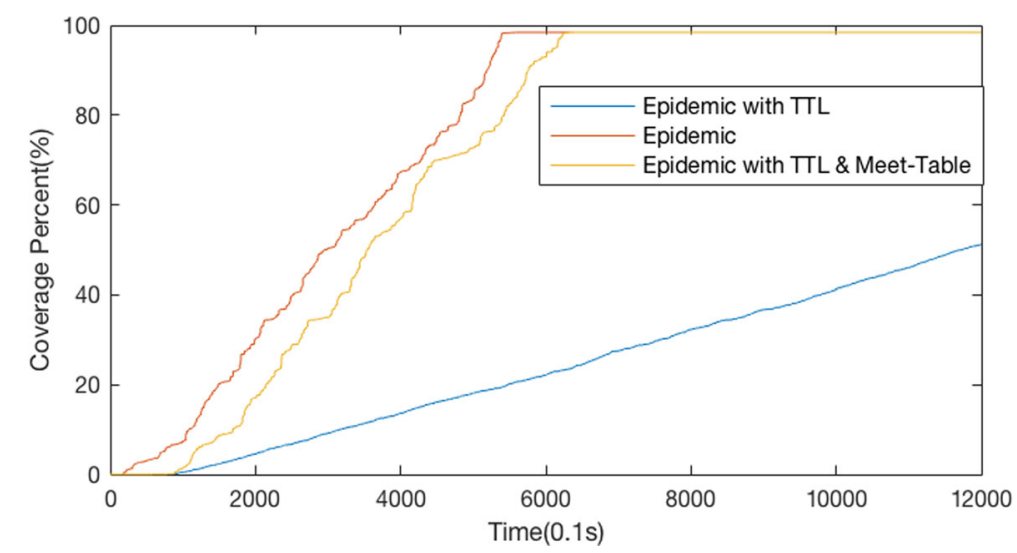

Fig. 4 Time vs. coverage percentage

position of obstacles, speed limit, and road accidents [2]. With the development of VANET, especially when selfdriving cars run on the road, security of VANET will be the key to safe traffic. Secure VANET requires ID authentication, message integrity, communication confidentiality, availability, and access control [12].

To satisfy these requirements, lots of solutions have been proposed. In these solutions, public key cryptography, trust management, blacklist, etc. are employed. Therefore, secure VANET needs to process messages about security in a secure and efficient way. As elsewhere, certificates used in VANET must be revoked in circumstances, such as compromising or losing of the private key and illegal using of the certificate [13]. CA (certificate authority) can issue CRL and store it on LDAP (Lightweight Directory Access Protocol) server for retrieving [14]. A vehicle can also use OCSP (Online Certificate Status Protocol) to request CRL [15]. Instead of directly accessing the Internet, a vehicle in VANET often access the Internet through infrastructure domain, so both to retrieve CRL from LDAP server and to request CRL using OCSP is not applicable.

VII (vehicle infrastructure integration) tries to distribute CRL to the vehicle through RSU broadcasting [5]. This method required a very large number of RSUs and high cost. In [6], Haas et al. try to propagate CRL in an epidemic fashion. The epidemic method can distribute CRL to all vehicles with less number of RSUs and less time, but it requires large storage and communication capacity in VANET.

Uncontrolled epidemic may cause flooding storm, so TTL is often used. For example, TTL is used to restrict the number of message replicas that a node is allowed to spread [8]. In [16], TTL is used to limit the HopTree update range to avoid over-overlapping of paths. But in VANET, a large TTL can hardly restrict flooding, while a small TTL will sharply decrease the coverage of messages.

In [9] mobile phone users' trajectory proves that humans follow simple reproducible patterns. According

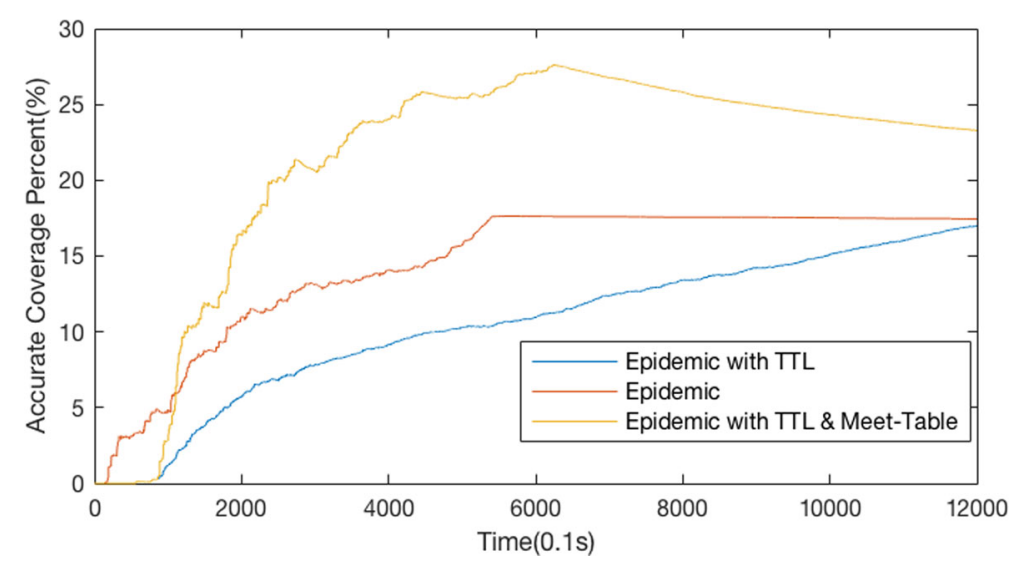

Fig. 5 Time vs. accurate coverage percentage 


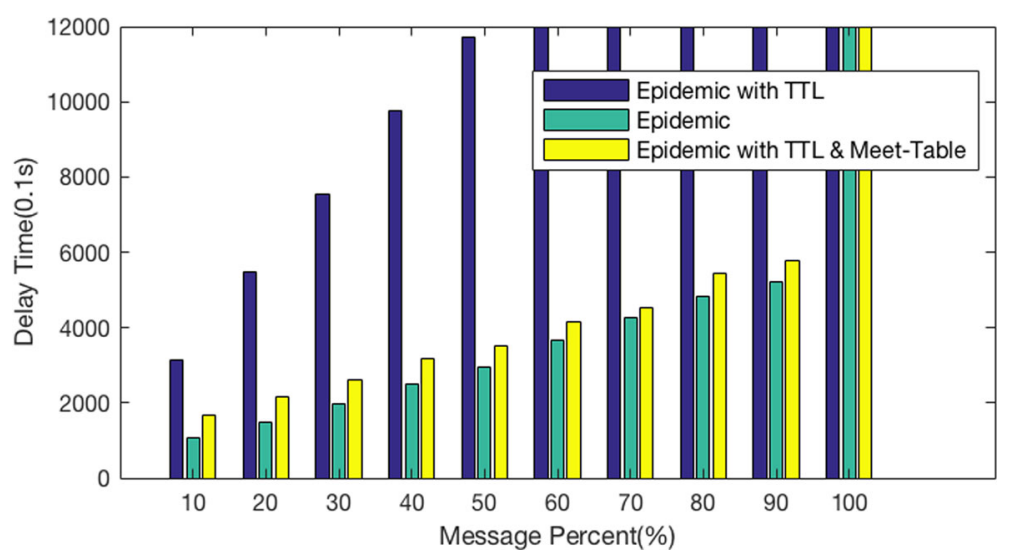

Fig. 6 Message percent vs. time delay

to the research results in $[10,17,18]$, VANET is a small world. In [19], a query processing algorithm that can determine the scope of each query is used to help vehicles to avoid returning overwhelmed large amount results. These works give us a clue to accurately distribute messages in VANET. Our previous work proposes Meet-Table to optimize CRL propagation in VANET [7]. And another work of us proposes the concept of a negative message and give a scheme based on Meet-Table and cloud computing to distribute negative messages in VANET [4].

This paper utilizes Meet-Table to lead TTL resetting in epidemic with TTL to overcome the shortage of it. It is a valuable advancement of our work on Meet-Table-based negative message management in VANET.

\section{Conclusions}

Negative messages play a crucial role in VANET security. Accurately and efficiently distributing negative messages is essential to make VANET safe against attacks. The epidemic method can rapidly distribute message but may cause flooding storm. The epidemic with TTL can control flooding storm but may sharply decrease coverage of negative messages in VANET. Meet-Table of a vehicle records vehicles it met, meaning the high probability that it will encounter these vehicles. Therefore, we utilize Meet-Table to lead TTL resetting in negative messages broadcasting. Simulation results of epidemic with and without TTL and epidemic with Meet-Table and TTL show that using MeetTable with TTL for the broadcasting of negative messages

Table 2 Summary of performance simulation results

\begin{tabular}{lllll}
\hline Method name & $r_{p}$ & $r_{c}$ & $r_{a c}$ & Delay \\
\hline Epdemic & High & High & Mid & Low \\
Epdemic with TTL & Low & Low & Low & High \\
Epdemic with TTL and Meet-Table & Mid & High & High & Low \\
\hline
\end{tabular}

has high coverage percentage, high accurate coverage percentage, and low delay of time. So, Meet-Table with TTL can not only increase coverage but also accurately and timely increase coverage.

In the future work, we will apply Meet-Table in VFC (vehicular fog computing) [20] to optimize bandwidth, storage, and computing requirements in VANET.

\section{Abbreviations}

AU: Application unit; CRL: Certificate revocation list; LDAP: Lightweight Directory Access Protocol; OCSP: Online Certificate Status Protocol; OBU: On-board unit; RSU: Roadside unit; TTL: Time-to-live; VANET: Vehicular ad hoc network; VFC: Vehicular fog computing; VIl: Vehicle infrastructure integration

\section{Funding}

This work was supported by the National Natural Science Foundation of China under Grant No. 61262072.

\section{Availability of data and materials}

Data sharing is not applicable to this article as no datasets were generated or analyzed during the current study.

\section{Authors' contributions}

$\mathrm{BH}$ proposed the idea of using Meet-Table with TTL to improve negative message broadcasting in VANET. XC formally defined the problem and wrote this paper. JM implemented the simulation. All authors read and approved the final manuscript.

\section{Authors' information}

Xiaolu Cheng is a Ph.D student at Virginia Commonwealth University. Baohua Huang, who visited Virginia Commonwealth University and worked with Xiaolu Cheng for 2 years from September 2015 to August 2017, is an associate professor of Guangxi University. Jiawei Mo is a postgraduate student of Baohua Huang at Guangxi University.

\section{Competing interests}

The authors declare that they have no competing interests.

\section{Publisher's Note}

Springer Nature remains neutral with regard to jurisdictional claims in published maps and institutional affiliations.

\section{Author details}

${ }^{1}$ School of Computer and Electronic Information, Guangxi University, 100 Daxue Road, Nanning 530004, Guangxi, China. ${ }^{2}$ Department of Computer Science, Virginia Commonwealth University, 401 West Main Street, Richmond 23220, VA, USA 
Received: 26 March 2018 Accepted: 28 August 2018

Published online: 12 September 2018

\section{References}

1. S. Al-Sultan, M. M. Al-Doori, A. H. Al-Bayatti, H. Zedan, A comprehensive survey on vehicular ad hoc network. J. Netw. Comput. Appl. 37(2), 380-392 (2014)

2. M. S. Kakkasageri, S. S. Manvi, Information management in vehicular ad hoc networks: a review. J. Netw. Comput. Appl. 39(3), 334-350 (2014)

3. H. Zhu, M. Li, L. Fu, G. Xue, Y. Zhu, L. M. Ni, Impact of traffic influxes: revealing exponential intercontact time in urban vanets. IEEE Trans. Parallel Distrib. Syst. 22(8), 1258-1266 (2011)

4. B. Huang, W. Cheng, in the 12th International Conference on Wireless Algorithms, Systems, and Applications. Distributing negative messages in VANET based on Meet-Table and cloud computing (Springer, Guilin, 2017), pp. 653-664

5. F. PB, Vehicle infrastructure integration-VII architecture and functional requirements, v1.1 (2010). http://ral.ucar.edu/projects/vii.old/vii/docs/ VIIArchandFuncRequirements.pdf. Accessed 1 Sept 2018

6. J. J. Haas, Y.-C. Hu, K. P. Laberteaux, Efficient certificate revocation list organization and distribution. IEEE J. Sel. Areas Commun. 29(3), 595-604 (2011)

7. B. Huang, J. Mo, Q. Lu, W. Cheng, in the 4th International Workshop on Network Optimization and Performance Evaluation. Optimizing propagation network of certificate revocation in VANET with Meet-Table (Springer, Zhangjiajie, 2016), pp. 147-154

8. S. Batabyal, P. Bhaumik, in 1st International Conference on Applications and Innovations in Mobile Computing, AIMoC 2014, February 27, 2014 - March 1, 2014. Proceedings - International Conference on 2014 Applications and Innovations in Mobile Computing, AIMoC 2014. Delay-overhead trade-offs in mobile opportunistic network using TTL based restricted flooding (IEEE Computer Society, Kolkata, 2014), pp. 9-14. http://doi.org/10.1109/ AIMOC.2014.6785512

9. M. C. Gonzalez, C. A. Hidalgo, A.-L. Barabasi, Understanding individual human mobility patterns. Nature. 453(7196), 779-782 (2008)

10. J. Ding, J. Gao, H. Xiong, in 23rd SIGSPATIAL International Conference on Advances in Geographic Information Systems. Understanding and modelling information dissemination patterns in vehicle-to-vehicle networks (ACM, New York, 2015), pp. 1-10

11. V. Naumov, R. Baumann, T. Gross, in 7th ACM International Symposium on Mobile Ad Hoc Networking and Computing. An evaluation of inter-vehicle ad hoc networks based on realistic vehicular traces (ACM, Florence, 2006), pp. 595-604

12. R. G. Engoulou, M. Bellaïche, S. Pierre, A. Quintero, VANET security surveys. Comput. Commun. 44(2), 1-13 (2014)

13. S. Chokhani, Toward a national public key infrastructure. IEEE Commun. Mag. 32(9), 70-74 (1994)

14. Y.-S. Yeh, W.-S. Lai, C.-J. Cheng, Applying lightweight directory access protocol service on session certification authority. Comput. Netw. 38(5), 675-692 (2002)

15. T. P. Hormann, K. Wrona, S. Holtmanns, Evaluation of certificate validation mechanisms. Comput. Commun. 29(3), 291-305 (2006)

16. D. N. Rewadkar, M. P. Madhukar, in 2014 2nd International Conference on Current Trends in Engineering and Technology, ICCTET 2014, July 8, 2014. 2nd International Conference on Current Trends in Engineering and Technology, ICCTET 2014. An adaptive routing algorithm using dynamic TTL for data aggregation in wireless sensor network (Institute of Electrical and Electronics Engineers Inc., Coimbatore, 2014), pp. 192-197. http://doi.org/ 10.1109/ICCTET.2014.6966286

17. F. D. Cunha, A. C. Vianna, R. A. F. Mini, A. A. F. Loureiro, in IEEE Conference on Computer Communications Workshops. Are vehicular networks small world? (IEEE, Toronto, 2014), pp. 195-196

18. H. Zhang, J. Lia, Modeling and dynamical topology properties of VANET based on complex networks theory. AIP Adv. 5(1), 1-11 (2015)

19. X. Wang, L. Guo, C. Ai, J. Li, Z. Cai, in 8th International Conference on Wireless Algorithms, System, and Applications (WASA). An urban area-oriented traffic information query strategy in VANETs (Springer, Zhangjiajie, 2013), pp. 313-324

20. V. G. Menon, Moving from vehicular cloud computing to vehicular fog computing: issues and challenges. Int. J. Comput. Sci. Eng. 9(2), 14-18 (2017)
21. SFMTA, San Francisco Transportation Fact Sheet (2013). https://www. sfmta.com/sites/default/files/FactSheet12-30-13_updated.pdf. Accessed 1 Sept 2018

22. W. Reisman, Commute speeds have slowed down for San Francisco drivers (2011). http://archives.sfexaminer.com/sanfrancisco/commutespeeds-have-slowed-down-for-san-francisco-drivers/Content?oid= 2187521. Accessed 1 Sept 2018

\section{Submit your manuscript to a SpringerOpen ${ }^{\circ}$ journal and benefit from:}

- Convenient online submission

- Rigorous peer review

- Open access: articles freely available online

- High visibility within the field

- Retaining the copyright to your article

Submit your next manuscript at $\boldsymbol{\triangleright}$ springeropen.com 